This generator, which has been most successfully used in a number of investigations, has several advantages over the known types: it is of simple construction and operation, and requires only a few minutes to reach stationary conditions. It produces larger quantities of aerosol than other apparatus and higher particle concentrations (up to $101 / \mathrm{min}$ of homogeneous aerosol and $10^{7}$ particles per $\mathrm{cm}^{3}$ ). Even substances which tend to decompose on prolonged heating can easily be converted into aerosols (e.g. D.O.P.), and the results are, within limits, reproducible (see Table I).

This investigation was sponsored by the Scientific Department, Isracli Ministry of Defence, and is published with its permission. We wish to thank Mr. R. Aloor for carrying out the measurements and calculations of the particle radii.

\section{E. RAPAPoRT and S. E. Weinstock}

Ministry of Defence, Scientific Department, Tel-Aviv, Israel, May 28, 7955.

\section{Zusammenfassung}

Es wird ein einfacher neuer Generator für homogene Aerosole beschrieben, welcher grössere Mengen und höhere Konzentrationen, als bisher bekannt sind, liefert.

Der Apparat erzeugt homogene Aerosole auch von Substanzen, welche nicht hitzebeständig sind.

\title{
Nouveaux livres - Buchbesprechungen - Recensioni - Reviews
}

\section{The Design and Analysis of Industrial Experiments}

By Owen L. Davies

637 pages

(Oliver and Boyd, Edinburgh, 1954)

$$
(63 /-)
$$

Dieses Buch, von einem Team von Chemikern und Statistikern geschrieben, wendet sich in erster Linie an den Forscher in der chemischen und pharmazeutischen Industrie. Planung, Durchführung und Auswertung von Experimenten werden darin vom Standpunkt der statistischen Methoden aus dargelegt, wobei der Nachdruck auf der Planung der Versuche unter besonderer Berücksichtigung ihrer Wirtschaftlichkeit liegt.

Die Ergebnisse chemischer Versuche pflegen häufig in aufeinanderfolgenden Serien anzufallen, und es ist dann wichtig, die ganze Versuchsfolge so anzuordnen, dass Ergebnisse früherer Versuche bei der Bestimmung von Richtung und eventuell Umfang weiterer Versuche möglichst erschöpfend berïcksichtigt werden. Im Anschluss an die Darstellung statistischer Tests im allgemeinen, die hauptsächlich vom Standpunkte der Versuchsplanung und unter Beriicksichtigung der mit den Tests verbundenen Risiken erfolgt, wird daher ausführlich auf die sogenannten Folgetests eingegangen.

Unter den eigentlichen Versuchsplänen werden zunächst zufällige Anordnungen in Blöcken, lateinische und griechisch-lateinische Quadrate und die verschiedenen Varianten der Anordnung in unvollständigen Blökken behandelt. Eine besonders eingehende Darstellung wird den Versuchen mit mehreren Faktoren, dem vollständigen und teilweisen Vermengen von Wechselwirkungen mit Unterschieden zwischen den Blöcken und den teilweise wiederholten Versuchen zuteil.

Die Versuche mit mehreren Faktoren leiten über zu den Verfahren, welche die Bestimmung der optimalen Arbeitsbedingungen in chemischen Prozessen zum Gegenstand haben. Diese Methoden zählen zu den neuesten der Statistik und dürften im Rahmen eines Lehr-oder Handbuches bisher kaum dargestellt worden sein. Besonders begrüssen wird es der Chemiker, dass er hier auch gleich die mathematischen Hilfsmittel und, im Hinblick auf die Anwendungen, die entsprechenden numerischen Methoden, deren Verwendung in diesem Zusammenhang nicht ganz umgangen werden kann, auseinandergesetzt findet.

Es gibt zahlreiche quantitativ-chemische Fragen, $\mathrm{zu}$ deren Lösung die Anwendung statistischer Verfahren den einzig methodisch einwandfreien und logisch sauberen Weg darstellt. Die statistische Seite mancher chemisch-technologischer Probleme zu erkennen, die Fragestellung entsprechend zu formulieren und selber die Methoden anzuwenden, dazu möchte das Buch den Chemiker in der Industrie anleiten. In der Stoffauswahl sind die Verfasser bestrebt, das für den Chemiker Wichtige in den Vordergrund zu stellen. Anderseits ist die Darstellung genügend allgemein gehalten, dass sie auch Ingenieure und Techniker verwandter Disziplinen interessieren dürfte. Die wesentlichen Ideen werden klar zum Ausdruck gebracht und, um die Entwicklung prinzipieller Fragen nicht $z u$ unterbrechen, findet die Begründung einzelner Verfahren und die Diskussion spezieller Gegenstände in den Anhängen zu den einzelnen Kapiteln Platz, welche etwa den dritten Teil des Buches einnehmen.

Das Buch ist klar und leichtfasslich geschrieben. Die mathematischen Kenntnisse, die es voraussetzt, sind recht bescheiden und die Darstellung bleibt immer anschaulich und direkt. Wertvoll sind vor allem die etwa fünfzig ausführlichen Beispiele, die dem Gebiet der chemischen Technologie und verwandten Gebieten entstammen. Ein Glossar statistischer Fachausdrücke und zahlreiche Tabellen samt Erläuterungen sowie ein ausführliches Sachregister beschliessen dieses Werk, das eine wertvolle Bereicherung nicht nur der statistischen, sondern auch der technischen Literatur darstellt. Wir zweifeln nicht daran, dass es die Anwendung statistischer Methoden in der Industrie erheblich fördern wird.

A. LINDER 